\title{
Synchrotron sheds new light on research questions
}

$\mathrm{T}$

en years ago, maybe one scientist was using the Canadian Light Source (CLS) synchrotron at the University of Saskatchewan for biomedical imaging. "It became two when I came here," says Dean Chapman, a specialist in the medical and biological applications of synchrotron light.

Since then an estimated 2500 researchers and scientists have lined up to use the synchrotron, which is a light millions of times brighter than the sun, 180 metres in diameter with 360 degrees of light and 18 "beamlines" for viewing samples at a molecular level. The fact that it's also big enough to handle large animals such as horses has researchers from around the world vying to use the facility for a veritable laundry list of research projects.

"We've imaged a dog as part of a prostate cancer project and a pig in a cystic fibrosis project," says Chapman, beamline team leader for Biomedical Imaging and Therapy. "We've imaged an early Bronze Age jaw bone from Siberia that has a spear point buried in it. We've imaged water formation in fuel cells.'

The synchrotron uses radio frequency waves and powerful electromagnets to accelerate electrons to nearly the speed of light. As the electrons accelerate, energy is added so that when the magnets alter their course they emit a brilliant, highly focused light. Different spectra of light, such as infrared, ultraviolet and X-rays, are directed down beamlines where researchers choose the desired wavelength to study their samples.

There are about 30 synchrotrons worldwide, but this one has a couple of unique niches. "We can image things in

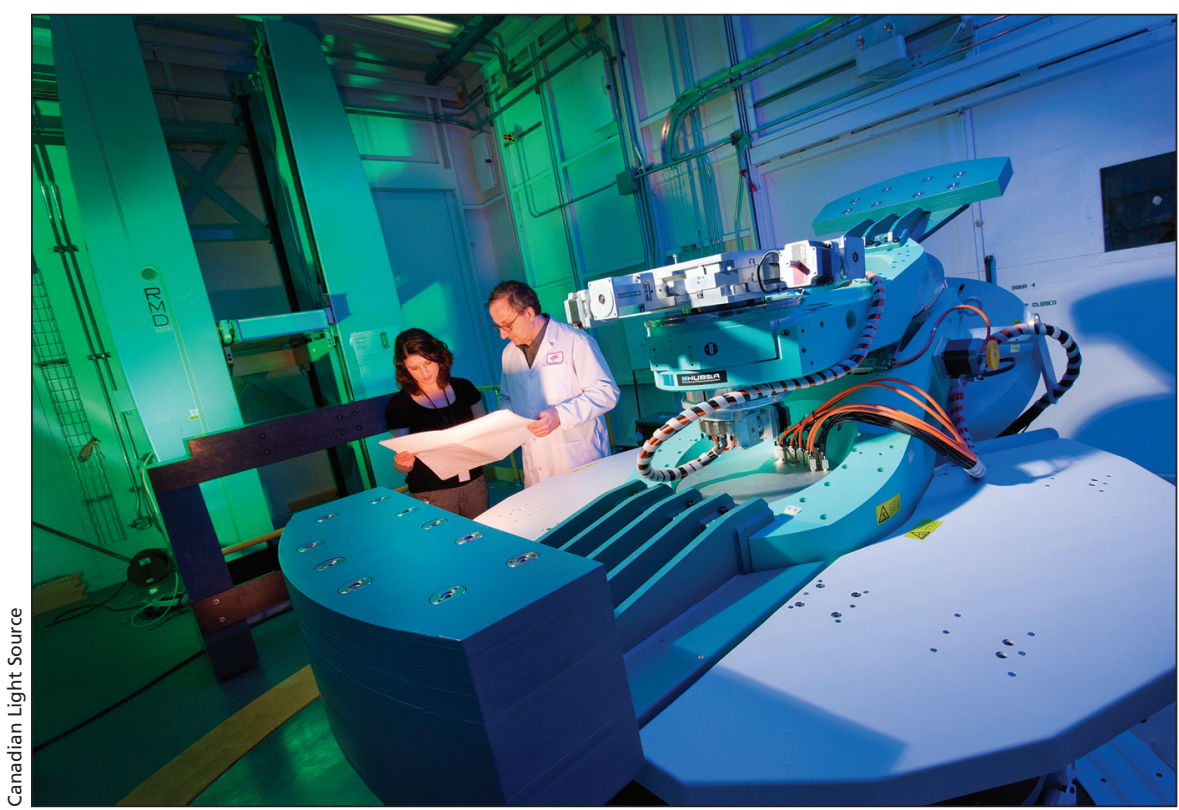

Scientists George Belev and Denise Miller at the biomedical imaging and therapy largeanimal positioning facility.

ways that would be very difficult if not impossible to do any other way," says Chapman, who holds the Canada Research Chair in X-ray Imaging. "We get very good soft tissue contrast at high resolution, something you don't normally think X-rays are capable of getting."

He says the CLS synchrotron imaging also specializes in tissue density. "Sometimes that's an advantage. Cancer for example, is a denser tissue. We can see that property. So most of the imaging is using that ability to see soft tissue in ways you normally wouldn't be able to."

Scientists have used CLS synchrotron results to help design new drugs, develop safer medical implants and analyze a host of physical, chemical, geological, and biological processes. Ten years in, Chapman says they are still discovering new applications.

"I don't think it has even been realized yet what you can do. Ten years sounds like a long time, but at least half of that was spent building the thing. And part of that time was spent growing a community."

Areas of medical research using the CLS synchrotron imaging include cystic fibrosis, Clostridium difficile, blood, heart arrhythmia, stroke and prostate cancer. Research projects accepted by the CLS synchrotron are chosen by an international peer-reviewed committee and ranked according to score. Becky Rynor, Saskatoon, Sask.

CMAJ 2015. DOI:10.1503/cmaj.109-5097 